\title{
Bioavailability of carotenoids in human subjects
}

\author{
Robert S. Parker*, Joy E. Swanson, Cha-Sook You, Alison J. Edwards and Tina Huang \\ Division of Nutritional Sciences, Cornell University, Ithaca, NY 14853, USA
}

\begin{abstract}
There is growing need for accurate information regarding the bioavailability of carotenoids, both with respect to carotenoids per se and to the vitamin A value of provitamin A carotenoids in foods or supplement preparations. Little quantitative information is currently available, owing primarily to the lack of adequate methods to assess carotenoid bioavailability. Methods applied to xenobiotic drugs are in most cases not useful for carotenoids, many of which circulate in appreciable quantities in human plasma. Reported ranges of carotenoid bioavailability (\% dose absorbed) range from 1-99, and variability is generally high both within and between treatments. With the current methods, relative bioavailability is more readily assessed than absolute bioavailability. The most commonly applied methods include measuring the increase in plasma carotenoid concentration following chronic intervention, and use of postprandial chylomicron (PPC) carotenoid or retinyl ester response following a single dose of carotenoid. The advantages and limitations of these approaches, together with examples of each, are discussed. A new PPC approach utilizing extrinsic-stable-isotope-labelled vitamin $\mathrm{A}\left({ }^{2} \mathrm{H}_{4}\right.$-labelled retinyl acetate) is under development in our laboratory, and examples of its application are presented. The currently available data suggest that oil solutions of carotenoids are more bioavailable than those from food matrices, and heating can improve the bioavailability of carotenoids from some food products. Increased availability of labelled carotenoids and retinoids should aid the development of reliable methods of carotenoid bioavailability assessment. Such data are needed for dietary recommendations, supplement formulation, and design of intervention strategies involving carotenoids.
\end{abstract}

Bioavailability: Carotenoids: Vitamin A: Postprandial chylomicron technique

Carotenoids are lipid-soluble plant pigments common in photosynthetic plants, animal tissues, and in the bloodstream and tissues of human subjects consuming plant and animal products. These pigments are of physiological interest in human nutrition, since some are vitamin A precursors and many exhibit radical or singlet oxygen trapping activity (Miller et al. 1996), and as such have potential antioxidant effects in vivo. Consequently, it is of value to determine the bioavailability of carotenoids from both foods and supplement forms in human subjects, and identify and quantify those factors which significantly affect carotenoid uptake and tissue disposition. Reliable data are needed to formulate effective policies for combating vitamin A deficiency and prevention of chronic degenerative diseases. At the present time there is considerable debate over such issues as the actual vitamin $A$ value of foods containing provitamin A carotenoids, and the relative importance of the large number of factors which may potentially influence carotenoid bioavailability. It is currently not possible to assign with confidence specific values to the absorption efficiency of a specific carotenoid from a specific food or preparation, nor is it possible to assign actual vitamin $\mathrm{A}$ values to specific plant foods consumed within a given meal or environmental context. Reported estimates of carotenoid absorption efficiency range from 1 to $99 \%$ ingested dose. Progress in this area has been hampered by the lack of adequate methodology to acquire this important information, particularly in human subjects. In many cases the debate has been confused by the use of various meanings of the term 'bioavailability'. This presentation will focus on key issues and problems related to the current state of knowledge of carotenoid bioavailability and its means of quantification. The discussion is not intended to be comprehensive, and the reader is directed to several recent reviews of various aspects of this topic (de Pee \& West, 1996; van Vliet, 1996; Parker, 1997). 
The concept of bioavailability as it relates to provitamin $A$ and non-provitamin A carotenoids

In the pharmaceutical arena the concept of bioavailability is well developed, and is commonly defined as the proportion of ingested drug which is absorbed and made available for its intended mode of action. The same concept has been applied to some nutrients with well-known biochemical functions and functional indicators, as in the case of riboflavin and erythrocyte glutathione reductase (EC 1.6.4.2) activity, or $\mathrm{Se}$ and glutathione peroxidase (EC 1.11.1.9) activity. In other cases the measured end point is a non-functional indicator of status, such as vitamin $A$ and plasma retinol, or Fe and transferrin saturation.

In this context, the issue of carotenoid bioavailability can be addressed in various ways, depending on whether the issue is the vitamin A value of the carotenoid, or the carotenoid per se. Operationally this distinction is important, since it will drive decisions related to the measured end point(s) and the method by which bioavailability is determined. Of course, for provitamin A carotenoids such as $\beta$-carotene, there may be interest in determining bioavailability from the standpoint of both vitamin $A$ value and the carotenoid per se. For the purpose of the present discussion, the term 'carotenoid bioavailability' will refer only to the carotenoid per se, without regard for any vitamin A activity the carotenoid may possess. The term 'vitamin A value' will be used to refer to the bioavailability of provitamin A carotenoids as it relates to their provitamin A function.

\section{Methods of assessing the bioavailability of carotenoids}

In the case of carotenoids, there is as yet no specific functional biochemical indicator of efficacy (analogous to riboflavin and erythrocyte glutathione reductase), or animal model of deficiency, other than the provitamin A activity of selected carotenoids. While carotenoids are hypothesized to be physiological antioxidants, they have not been shown to be active in the classical animal models of antioxidant deficiency, such as exudative diathesis in the chick induced by combination deficiency of vitamin $\mathrm{E}$ and Se (Mayne \& Parker, 1989). This does not mean that carotenoids are not antioxidants, rather that they do not substitute for the nutrients for which these models were developed, therefore what activity they may possess cannot be assessed by such models.

In the absence of a functional marker for biological activity of carotenoids in intact organisms, 'bioavailability' of carotenoids has largely been taken to mean 'assimilation efficiency', or the ability to accumulate them in some defined body pool. Such pools may be highly dynamic, such as plasma chylomicrons following a carotenoid-rich meal, total plasma or plasma lipoproteins following subchronic or chronic dietary supplementation, or more static pools such as adipose tissue or the macular pigment of the eye. The xanthophylls lutein and zeaxanthin together largely comprise the macular pigment of the human retina, implicating these carotenoids in macular function and prevention of macular degeneration (Bone et al. 1988). Thus, macular pigment density, which can be assessed non-invasively by physical methods to assess its relationship with carotenoid intake (Hammond et al. 1997), may emerge as a nearfunctional indicator of bioavailability of lutein or zeaxanthin. A comprehensive review of the relative merits of all potential end points is beyond the scope of the present paper, and only selected models will be discussed in detail.

\section{Bioavailability of carotenoids as assessed by absorption efficiency in human subjects}

The term 'absorption efficiency' is defined as the proportion of ingested carotenoid which is secreted into the general circulation and therefore made available for tissue uptake, with a common unit of expression being \% dose. Absolute absorption efficiency of xenobiotic drugs is commonly assessed by comparing area under the plasma concentration $v$. time curve of orally-administered drug with that obtained with intravenous administration, the latter taken as $100 \%$ absorption efficiency. Since most carotenoids of current interest already exist in plasma at appreciable concentrations, this approach is not useful. Consequently absolute absorption efficiency of carotenoids is difficult to measure without invoking various assumptions, such as the extent of faecal degradation in models using oral-faecal balance, or input and elimination rate-constants in the case of postprandial chylomicron (PCC) response models. Given the inherent difficulties in measurement of absolute absorption, some of which are discussed in detail later, it is not surprising that reported estimates have ranged from 1 to $99 \%$. An absorption efficiency of $33 \%$ is currently applied to the $6: 1$ mass equivalence of food-borne $\beta$-carotene and derived retinol. More conveniently assessed is 'relative bioavailability', where two or more treatments are compared.

As illustrated in Fig. 1, carotenoids are taken up by the mucosa of the small intestine, packaged into triacylglycerol-rich chylomicrons, and secreted into the lymph for delivery to the bloodstream where they are rapidly degraded by lipoprotein lipase (EC 3.1.1.34). The resulting chylomicron remnants are rapidly taken up by the liver (Parker, 1996). The liver secretes carotenoids associated with hepatic VLDL, and in the fasting state most plasma carotenoids are associated with LDL and HDL. Thus, newly-absorbed carotenoids will be found primarily in the chylomicron fraction. However, because of the rapid rate of chylomicron catabolism and hepatic uptake of chylomicron remnants (half-life 10-15 min), even in the postprandial state chylomicron-associated carotenoids represent only a small proportion of total plasma carotenoids.

For this reason whole plasma generally cannot be successfully used to quantify newly-absorbed carotenoids, with a few exceptions. Carotenoids can be labelled so as to distinguish them from the much larger LDL- and HDLcarotenoid pools. ${ }^{13} \mathrm{C}$ - and ${ }^{2} \mathrm{H}$-labelled $\beta$-carotene in pure form have been used to examine the kinetics of carotene uptake and removal from whole plasma (Parker et al. 1993, 1997; Novotny et al. 1995). Selected food products can be intrinsically labelled with ${ }^{2} \mathrm{H}$ by growing with ${ }^{2} \mathrm{H}_{2} \mathrm{O}$. It may also be possible to use whole plasma to study absorption of carotenoids for which baseline plasma concentrations are very low, such as astaxanthin, echininone, or other pigments 


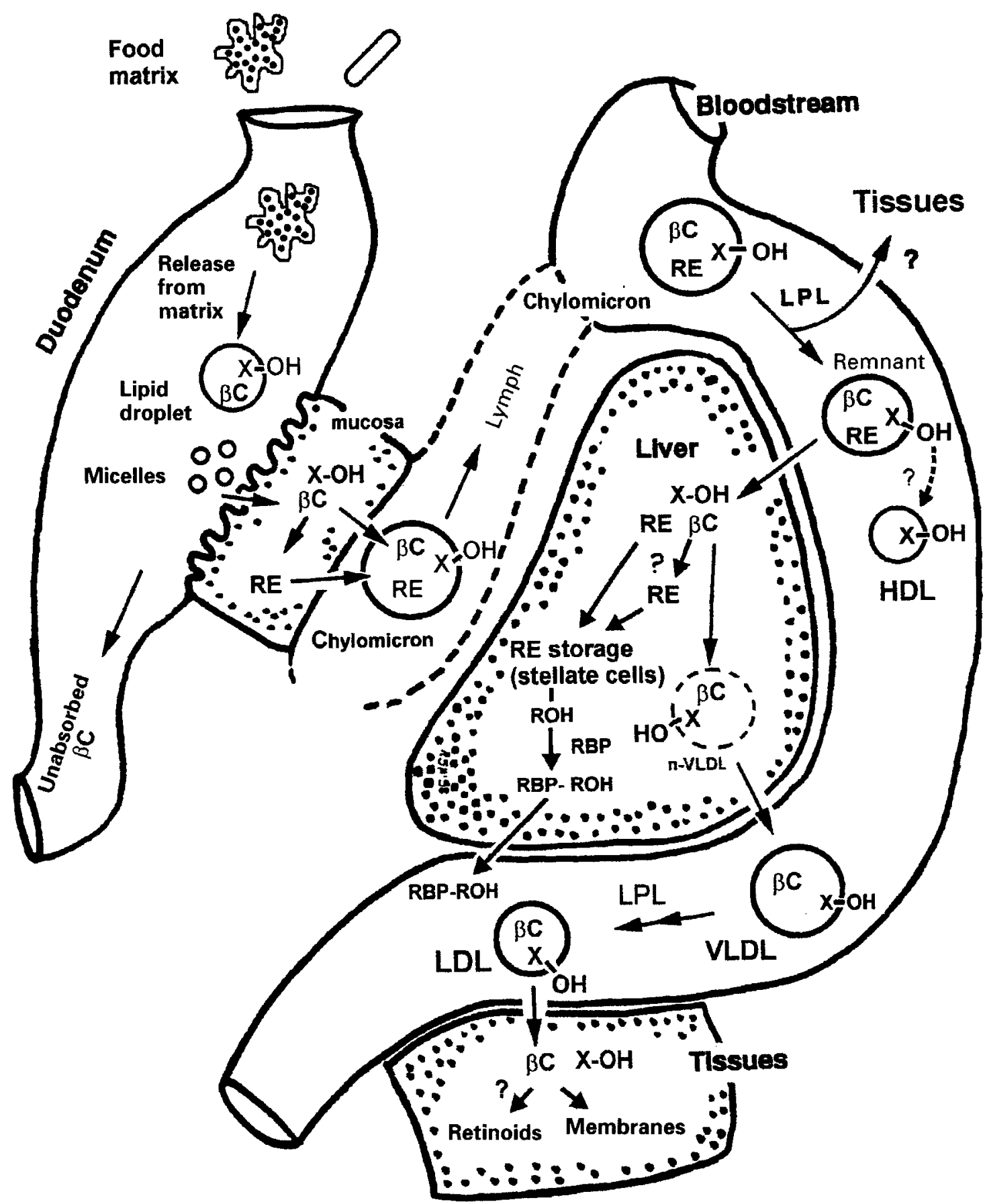

Fig. 1. Scheme for uptake, metabolism and transport of carotenoids. $\beta C, \beta$-carotene; $X-O H$, xanthophylls; RE, retinyl esters; RBP, retinol-binding protein; ROH, retinol; n-VLDL, nascent VLDL; LPL, lipoprotein lipase (EC 3.1.1.34).

which exist in human foodstuffs but which normally occur only in trace amounts in plasma.

Most carotenoids of current biomedical interest, such as $\beta$-carotene, lycopene, and lutein, circulate at significant concentrations in human plasma. Consequently, whole plasma cannot be used to accurately assess the extent of absorption of carotenoids from a test meal. Since chylomicron carotenoids represent newly-absorbed material, the so-called triacylglycerol-rich plasma fraction, defined by a density less than $1.006 \mathrm{~kg} /$, is potentially very useful in assessing bioavailability of carotenoids, at least as reflected by their absorption efficiency. This model, referred to here as the PPC model, was applied first to $\beta$-carotene by van Vliet et al. (1995), and since to other carotenoids (Gartner et al. 1997; O'Neill et al. 1998). This model is essentially a single-dose pharmacokinetic approach in which the end point is the carotenoid concentration in a plasma fraction enriched in chylomicron remnants. Typically, the carotenoid of interest is fed with a standard meal, and blood samples withdrawn before the meal and at 
various time intervals over several hours after the meal. The density $<1.006 \mathrm{~kg} / 1$ fraction is isolated by conventional ultracentrifugation for time periods varying from $30 \mathrm{~min}$ to $18 \mathrm{~h}$, and the concentration of carotenoid determined by HPLC. The mass of carotenoid absorbed is proportional to the area under the concentration $v$, time curve generated from the baseline-corrected data. Baseline carotenoid concentrations should typically be very low in the absence of contamination from other lipoprotein fractions, or abnormalities in the lipoprotein metabolism of the subject.

While currently one of the most useful means of assessing carotenoid absorption efficiency, the PPC model suffers from several pitfalls. Since newly-secreted chylomicrons undergo lipolysis by lipoprotein lipase immediately upon entering the bloodstream, the plasma chylomicron population in reality consists of remnants of a range of particle sizes and densities. The proportion of the remnant pool which is recovered will be a function of the chosen density and the period of centrifugation (30 min-14h) and speed. None of the PPC models published to date includes a means of assessing the recovery of chylomicrons in the collected (analysed) fraction, and it is highly likely that different centrifugation methods will result in greatly differing recoveries. This is particularly problematic for estimates of absolute absorption efficiency, as underrecovery will directly result in underestimation of absorption efficiency. It is likely that much of the disparity in reported area-under-curve data (for example, that of van Vliet et al. 1995; O'Neill et al. 1998) is due to differential (and unknown) recovery of chylomicron-associated carotenoids. The issue of recovery is less problematic in the case of assessment of relative bioavailability, since it need only be assumed that recovery is invariant from one sample to another when using the same procedure.

Another problem inherent to the PPC model, as it is currently applied, is that area-under-curve data are typically highly variable (several fold), especially between subjects, even though treatment conditions are usually standardized. This has led in some cases to the conclusion that carotenoid absorption is highly variable. While such variation may indeed occur, alternatively it may be a result of differences in kinetics of chylomicron secretion and clearance. The current PPC models assume constant rates of chylomicron secretion (plasma input) and hepatic remnant uptake (plasma elimination), both between subjects and between treatments. However, the instantaneous concentration of carotenoid in the plasma chylomicron (remnant) population will be a function of the concentration of carotenoid in the secreted particles, the rate of plasma input, and the rate of plasma elimination. While it is highly likely that different individuals exhibit different kinetics of particle input or removal, or that subjects exhibit different kinetics on different days, current PPC models contain no means of controlling for such differences. High variability can greatly impair the ability to discern differences between treatments. Nonetheless, the PPC model has been used successfully to obtain relative bioavailability information, such as the case of lycopene from raw $v$. heated tomato products (Gartner et al. 1997). Their findings support the suggestion that heating of plant food products can increase the bioavailability of at least some carotenoids, perhaps by weakening protein- carotenoid interactions, or dissolving and dispersing crystalline carotenoid deposits, or improving digestibility.

A third issue still unresolved is the extent to which the PPC model can be applied to xanthophylls. Unlike the hydrocarbon carotenes such as $\beta$-carotene and lycopene, xanthophylls possess polar functional groups. As such, xanthophylls will be largely surface components of the chylomicron (and other lipoproteins), while the carotenes will exist primarily in the core of the particles. Surface polar lipid constituents such as cholesterol, phospholipid, and the tocopherols are all known to exchange between lipoprotein particles during circulation. For example, $\alpha$-tocopherol rapidly appears in HDL during the early postprandial stage following an oral dose, a phenomenon which cannot be accounted for by liver secretion (Traber et al. 1994). Excess surface lipids resulting from lipoprotein lipase hydrolysis of chylomicron core triacylglycerols and subsequent particle shrinkage is thought to be the source of the newly-acquired HDL-tocopherol. A similar phenomenon may occur with xanthophylls such as lutein, and if so, PPC lutein concentrations may under-represent the true extent of lutein absorption. Current applications of the PPC model to compare absorption efficiency of different carotenoids assume equivalent recoveries regardless of chemical structure, but this assumption requires validation. Use of ${ }^{13} \mathrm{C}$ or ${ }^{2} \mathrm{H}$-labelled xanthophylls, or unlabelled xanthophylls which exist in trace amounts in human plasma, should prove useful for this purpose.

\section{Bioavailability of carotenoids as assessed by plasma carotenoid concentration}

Since carotenoids are absorbed by simple diffusion and lack specific plasma binding proteins, their concentration in plasma lipoproteins within individuals will to some extent reflect the rate of dietary intake. Dose-response studies have been conducted with $\beta$-carotene (for review, see Swanson \& Parker, 1996), but not with other purified carotenoids due to their lack of availability in sufficient quantities. Change from baseline of plasma carotenoid concentration can be useful as an end point to estimate the relative bioavailability of carotenoids in human subjects, provided such studies are sufficiently long to result in a new steady-state condition. Two examples of this approach are discussed here. Rock et al. (1998) used a 4-week crossover approach to examine the relative bioavailability of $\beta$-carotene in raw $\nu$. processed carrots (Dorcus carota) and spinach (Spinacia oleracea), and concluded that a combination of particle size reduction and heat treatment increased bioavailability of $\beta$-carotene from these commodities. Of course, this model does not account for potential treatment-related differences in efficiency of intestinal conversion of $\beta$-carotene to vitamin $A$.

We have recently compared the bioavailability of $\beta$ carotene from gelatin beadlets dispersed in yogurt as a function of yogurt milk fat content and sex. Healthy older males and females (55-80 years) consumed $\beta$-carotenefortified yogurts of either 0 (skim milk) or $10 \mathrm{~g} \mathrm{milk} \mathrm{fat} / \mathrm{kg}$ for 3 weeks in a crossover design, with plasma $\beta$-carotene change as the end point. Subjects were supplied with a standardized low-fat low-carotenoid diet during the study period, and yogurts supplying $30 \mathrm{mg} \beta$-carotene/d. As 


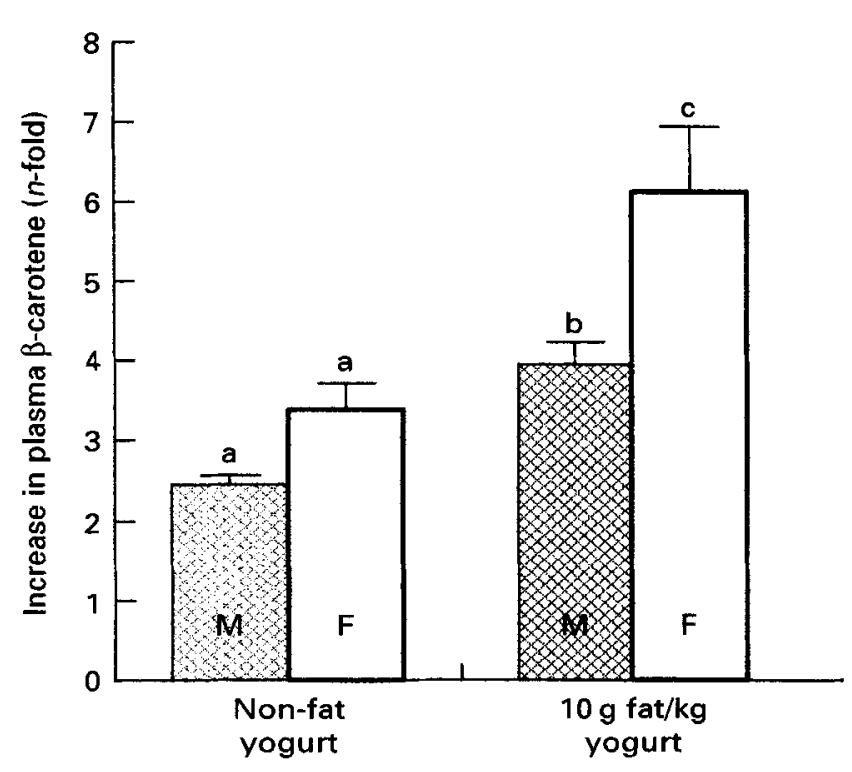

Fig. 2. Comparison of change in plasma $\beta$-carotene concentration in groups of healthy older male $(M)$ and female $(F)$ subjects $(55-80$ years) supplemented daily for 3 weeks with either non-fat or $10 \mathrm{~g}$ milk fat $/ \mathrm{kg}$ yogurt containing $30 \mathrm{mg} \beta$-carotene as gelatin beadlets. Values are means with their standard errors represented by vertical bars. $a, b, c$ Mean values with unlike superscript letters were significantly different $(P<0.05)$.

illustrated in Fig. 2, there was a significant effect of yogurt milk fat content, with the $10 \mathrm{~g}$ milk fat $/ \mathrm{kg}$ yogurt resulting in greater increases in plasma $\beta$-carotene levels than the non-fat yogurt. In addition, females exhibited a significantly greater response than males, regardless of yogurt milk fat content.

\section{Bioavailability of provitamin A carotenoids: vitamin $A$ value}

The previous discussion has dealt only with assimilation of carotenoids per se, without regard for potential metabolic biotransformation, either in the intestinal mucosa during absorption, or post-absorptively. For the non-provitamin A carotenoids, this is perhaps less of an issue. However, for the provitamin A carotenoids, the mass of carotenoid assimilated intact will be a function of not only the efficiency of uptake into the intestinal mucosa, but the extent of metabolism of mucosal carotenoid to vitamin A. Thus, inferences regarding 'absorption efficiency' of provitamin A carotenoids must often take into account both the carotenoid and the yield of vitamin A in order to avoid underestimation of total percentage of the dose assimilated.

The major dietary provitamin $A$ carotenoids are $\beta$ carotene, $\alpha$-carotene and cryptoxanthin. It is commonly assumed that $50 \%$ of such carotenoids taken up by the intestinal mucosa undergo metabolism to ultimately yield retinyl esters, which are secreted along with surviving carotenoids in chylomicrons. Current findings support central oxidation of $\beta$-carotene to yield two molecules of vitamin A (for review, see Parker, 1997). Other provitamin A carotenoids apparently yield $1 \mathrm{~mol}$ vitamin A/mol carotenoid undergoing metabolism. The post-absorptive metabolic fate of carotenoids, including those with vitamin A value, is very poorly understood, but it is generally considered that the majority of carotenoid-derived vitamin $\mathrm{A}$ is of intestinal origin.

Vitamin A produced via metabolism of provitamin A carotenoids is absorbed exclusively in the form of retinyl esters associated with chylomicrons. The liver does not secrete retinyl esters, except as a consequence of high chronic doses of preformed vitamin A, which can result in saturation of the storage capacity of the liver (Olson, 1987). Consequently, it is theoretically possible to assess the intestinal vitamin A yield of provitamin A carotenoids by measuring the retinyl ester response in postprandial whole plasma. However, in practice this is generally not feasible because of the low instantaneous concentration of chylomicron retinyl ester, the relatively low sensitivity of direct determination of retinyl ester concentration by HPLC, and the presence of large quantities of other lipids in extracted plasma. The latter cannot be removed by saponification since the resulting free retinol would mix with extracted retinol associated with retinol-binding protein, which far exceeds the concentration of chylomicron retinyl esters. Consequently, the PPC approach is currently one of the few methods for assessing vitamin A yield of provitamin A carotenoids, and has the advantage that information on intact carotenoids can be obtained as well. To date this approach has been applied to the study of intestinal uptake and metabolism of $\beta$-carotene (van Vliet et al. 1995; O'Neill et al. 1998), but not to other provitamin A carotenoids.

Use of the PPC approach to determine absolute vitamin A value (mass of retinyl ester assimilated per unit mass of carotenoid consumed) has been hampered by the same pitfalls discussed previously, i.e. lack of internal controls for chylomicron recovery and kinetics. Consequently, it is not surprising that the available data are highly variable, especially between studies. For example, retinyl ester and $\beta$ carotene area-under-curve values following administration of $15 \mathrm{mg} \beta$-carotene as gelatin beadlets (van Vliet $e t$ al. $1995)$ were higher than those following administration of $40 \mathrm{mg} \beta$-carotene in the form of red palm oil (O'Neill et al. 1998), even though both were fed with ample lipid. This discrepancy may well be accounted for by differential chylomicron recovery between the two studies, which employed different centrifugation methods.

Our laboratory has recently developed a modification of the PPC approach which may avoid some of the pitfalls related to variability in chylomicron recovery and kinetics. This approach involves use of an extrinsic label, ${ }^{2} \mathrm{H}_{4}$ labelled retinyl acetate, fed along with the test meal. Preformed vitamin A is generally considered to be absorbed with relatively high efficiency, and is relatively refractory to conditions which may significantly affect carotenoid absorption, such as meal fat or fibre content (Olson, 1987). Retinyl acetate, like other dietary retinyl esters, is hydrolysed in the intestine to retinol, which is then taken up either by diffusion with lipid micelles or by a proposed facilitated transport mechanism (Ong, 1994). Once in the mucosa the retinol is esterified and secreted with chylomicrons, along with carotenoids and carotenoid-derived retinyl esters. If one assigns a constant absorption efficiency to the extrinsic 
${ }^{2} \mathrm{H}_{4}$-labelled retinyl acetate (e.g. $80 \%$ of the dose), and further assumes that the absorbed ${ }^{2} \mathbf{H}_{4}$-labelled retinyl esters are secreted and cleared with approximately the same kinetics as the fed carotenoids and unlabelled retinyl ester derived from them, the extrinsic label becomes essentially an internal standard controlling for both chylomicron recovery and kinetics. HPLC is used to quantify carotenoids appearing in the saponified density $<1.006 \mathrm{~kg} / 1$ plasma fraction prepared using $14 \mathrm{~h}$ ultracentrifugation at $140000 \mathrm{~g}$, and employing appropriate internal standards. GC-mass spectrometry is used to differentiate between labelled and unlabelled retinol in this fraction. Details of this method are being published separately.

We are using this approach to study the absorption and vitamin $\mathrm{A}$ yield of foods containing provitamin A carotenoids such as carrot and spinach. Fig. 3 illustrates typical PPC response curves of ${ }^{2} \mathrm{H}_{4}$-labelled retinyl ester, unlabelled retinyl ester, $\beta$-carotene, and $\alpha$-carotene in a subject fed on raw carrot (containing $9.8 \mu \mathrm{mol} \beta$-carotene and $5.2 \mu \mathrm{mol} \alpha$-carotene) and $2 \mathrm{mg}(7 \mu \mathrm{mol}){ }^{2} \mathrm{H}_{4}$-labelled retinyl acetate in a test meal with $20 \mathrm{~g}$ fat. The subject was given a simple midday meal, also containing $20 \mathrm{~g}$ fat, but no sources of vitamin A or carotenoid, $3 \mathrm{~h}$ after the test meal. The kinetics of the labelled and unlabelled retinyl ester and the carotenoids were similar, indicating that the extrinsic label mixed with the carotenoid and retinyl ester pool derived from the carrot. Assuming $80 \%$ absorption efficiency for the extrinsic ${ }^{2} \mathrm{H}_{4}$-labelled retinyl acetate, the area-under-curve values for the four analytes are equivalent to $5.6 \mu \mathrm{mol}{ }^{2} \mathrm{H}_{4}$-labelled retinyl ester, $1.5 \mu \mathrm{mol}$ unlabelled retinyl ester, $0.8 \mu \mathrm{mol} \beta$-carotene, and $0.4 \mu \mathrm{mol} \alpha$-carotene. Based on the $9.8 \mu \mathrm{mol} \beta$-carotene fed as carrot, the $0.8 \mu \mathrm{mol}$ $\beta$-carotene estimated to have been absorbed intact represented about $8 \%$ of the dose. Assuming $2 \mathrm{~mol}$ retinyl ester per mol $\beta$-carotene converted, the total $\beta$-carotene

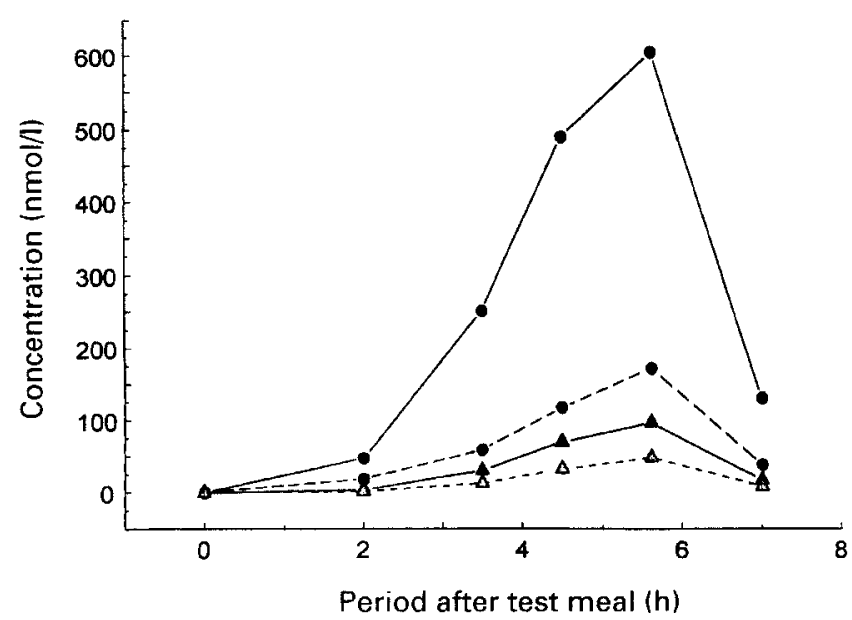

Fig. 3. Kinetics of ${ }^{2} \mathrm{H}_{4}$-labelled retinyl ester (-) , unlabelled

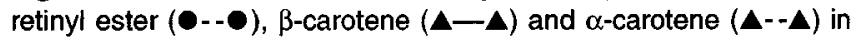
the postprandial chylomicron fraction of a subject given a test meal containing $5.25 \mathrm{mg}(9.8 \mu \mathrm{mol}) \beta$-carotene as raw carrot plus $2 \mathrm{mg}$ $(7 \mu \mathrm{mol}){ }^{2} \mathrm{H}_{4}$-labelled retinyl acetate. Area-under-curve values were ( $\mu \mathrm{mol}):{ }^{2} \mathrm{H}_{4}$-labelled retinyl ester 5.6 (assigned by assuming constant absorption efficiency of $80 \%$ of the dose), unlabelled retinyl ester 1.5 , $\beta$-carotene $0.8, \alpha$-carotene 0.4 . uptake would be equivalent to $16 \%$ of the dose. This value is probably a slight overestimate, since the potential contribution of $\alpha$-carotene to the unlabelled retinyl ester peak is not taken into consideration. The expected retinyl ester yield, assuming the standard $6: 1 \beta$-carotene-vitamin A mass equivalency, would be $0.9 \mathrm{mg}$ retinol from the dose (as carrot) of $5 \mathrm{mg}$. The observed yield was $0.4 \mathrm{mg}$, or a mass equivalency of about $13: 1$. In actuality, this ratio may be low since the contribution of $\alpha$-carotene is unknown and not considered.

This extrinsic label approach may thus be used to estimate the absolute vitamin A value, as calculated in the previous example. Of course, the derived values are based on an assumed absorption efficiency of retinyl acetate. While generally considered to be absorbed with relatively high efficiency (Olson, 1987), the most appropriate value is uncertain (Blomhoff et al. 1991). Alternatively, it may be used simply to compare the vitamin A yield of a product with that of preformed vitamin $A$ under the prevailing meal situations by comparing the ${ }^{2} \mathrm{H}_{4}$-labelled retinyl ester and unlabelled retinyl ester area-under-curve values. The latter requires no assumptions regarding the absorption efficiency of the extrinsic label, rather inferences about the product are made relative to the 'best case'. In the previous example, the vitamin $A$ value of the mass of carrot fed could be viewed as being about $20 \%$ that of a $2 \mathrm{mg}$ dose of preformed vitamin $A$ under the conditions prevailing at the time of administration.

\section{Uptake and metabolism of carotenoids as saturable phenomena}

At physiological doses of $\beta$-carotene, or amounts reasonably expected from foods, the concentration of retinyl ester in the PPC fraction consistently exceeds that of $\beta$-carotene, indicating that conversion efficiency typically exceeds $50 \%$ (assuming $2 \mathrm{~mol}$ retinyl ester per mol $\beta$-carotene metabolized). This has been reported both with the PPC model (van Vliet et al. 1995) and with purified ${ }^{13} \mathrm{C}$-labelled $\beta$-carotene (Parker et al. 1997). However, at high doses of $\beta$-carotene it is possible to saturate the capacity of the intestine to convert $\beta$-carotene to retinyl esters, and a greater proportion of absorbed material is secreted as intact $\beta$-carotene. We have used ${ }^{13} \mathrm{C}$-labelled $\beta$-carotene and highprecision isotope-ratio mass spectrometry along with HPLC (Parker et al. 1997) to illustrate this point, as shown in Fig. 4. Subjects were given either $1 \mathrm{mg}$ labelled $\beta$-carotene or $1 \mathrm{mg}$ labelled $\beta$-carotene plus $49 \mathrm{mg}$ unlabelled $\beta$-carotene on separate occasions. Postprandial concentrations of total retinyl esters (labelled plus unlabelled) and $\beta$-carotene were determined in whole plasma. At the low dose, the mass of absorbed retinyl ester exceeded that of absorbed $\beta$-carotene, but at the high dose the situation was reversed. This indicates that conversion capacity had been exceeded at the higher $\beta$-carotene dose. Saturation was also apparent in the findings of O'Neill et al. (1998) who fed $40 \mathrm{mg} \beta$-carotene as red palm oil, such that virtually every subject absorbed more $\beta$-carotene than retinyl ester. Since mucosal uptake of carotenoids apparently occurs by simple diffusion, uptake is probably also saturable with administration of high doses of pure carotenoids in oil solution. Thus, when using 


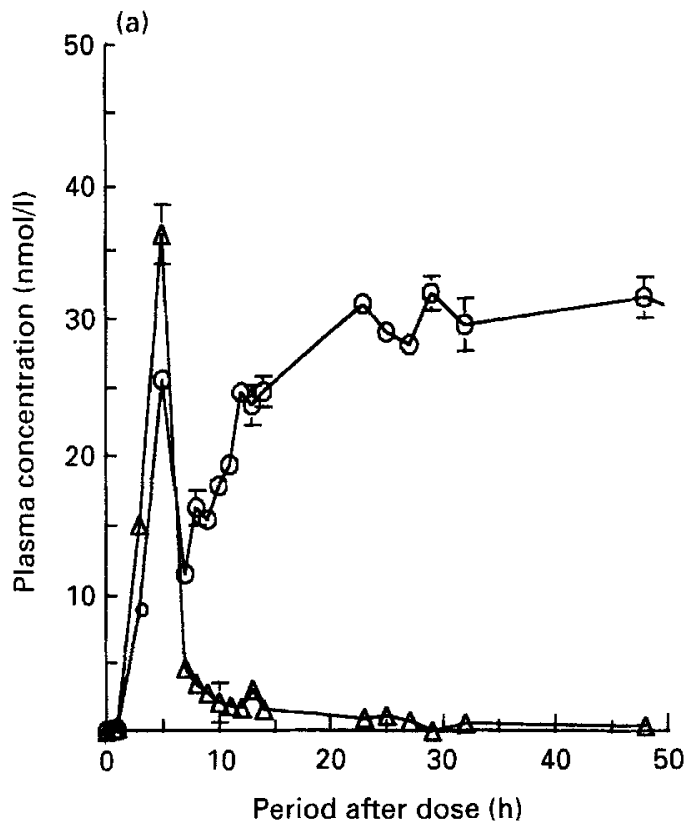

(b)

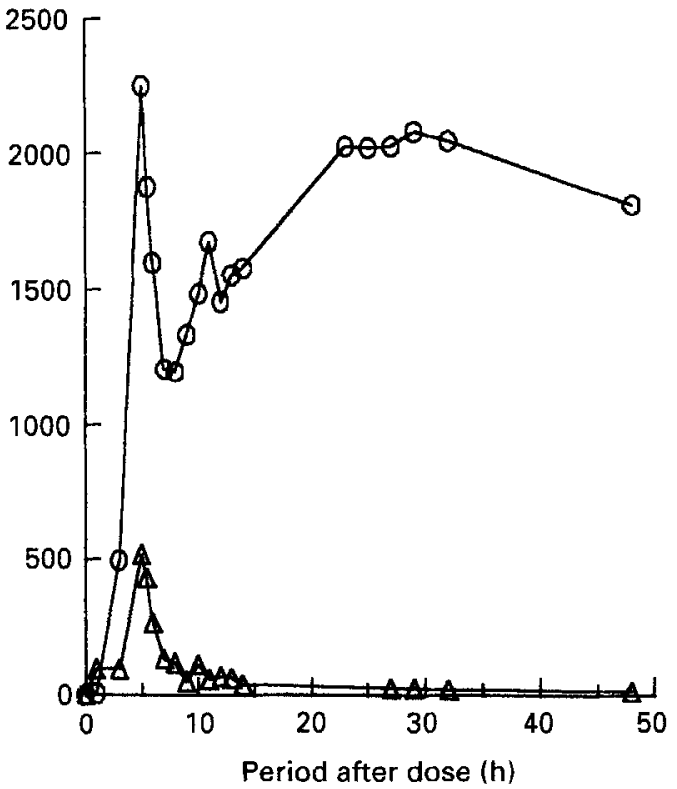

Fig. 4. Plasma kinetics of $\beta$-carotene $(O)$ and retinyl esters $(\triangle)$ following single doses of either (a) $1 \mathrm{mg}$ or (b) $50 \mathrm{mg}$ pure all-trans $\beta$-carotene dissolved in $20 \mathrm{~g}$ vegetable oil (i.e. $1 \mathrm{mg}{ }^{13} \mathrm{C}$-labelled $\beta$-carotene or $1 \mathrm{mg}{ }^{13} \mathrm{C}$-labelled $\beta$-carotene $+49 \mathrm{mg}$ unlabelled $\beta$-carotene).

carotenoid preparations which are likely to be highly bioavailable, one must use caution to avoid doses which approach or exceed saturation of either uptake or conversion.

The term 'bioavailability' should not be taken as synonymous with 'efficacy', as for example in the use of carotenoids to improve vitamin A status. The two terms are related, but efficacy can be affected by many factors other than bioavailability. For example, lactating women may exhibit elevated rates of utilization of vitamin $A$ due to its secretion in milk. A lack of effect of intervention with a given carotenoid, in the absence of the proper controls, may be more reflective of insufficient dose, rather than of low bioavailability of the treatment per se. It is perhaps useful to restrict the term bioavailability to include those factors which affect absorption efficiency (or intestinal metabolism), i.e. factors inherent to the dose formulation or to its digestion and intestinal processing. Thus, for example, dietary fibre or intestinal parasites may reduce bioavailability of carotenoids, while fever, pregnancy, or lactation influence efficacy. Assessment of total body vitamin A stores by isotope dilution may be very useful to evaluate the effectiveness of various interventions (Haskell et al. 1997), but since vitamin A stores are a function of both rate of assimilation and rate of utilization, the approach does yield information on bioavailability per se.

\section{Concluding remarks}

Accurate information on the bioavailability of carotenoids is needed in order to devise food-based strategies for longterm alleviation of vitamin A deficiency, and food or supplement-based interventions to reduce risk for chronic diseases which appear linked to carotenoid intake or blood levels. At the present time only generalities can be made, and information on specific commodities or products is lacking. Carotenoids from oil solutions appear to be more bioavailable than those from food matrices, although very few foods have been examined. Heating foods appears to increase carotenoid bioavailability, although important variables such as severity of heat or commodity-specific effects remain largely unknown. Progress in this area has been hampered by the lack of methods suitable for the reliable measurement of carotenoid bioavailability or vitamin A yield. Single-dose pharmacokinetic models based on chylomicron carotenoid or retinyl ester response are likely to be useful for evaluation of specific commodities and conditions in the context of single meals. Other models based on chronic treatment may be useful to discern more subtle or longer-term differences between treatments, and for field studies in target populations (e.g. young children) where pharmacokinetic studies are problematic. Thus, no single method is likely to serve all purposes. Rather, methods need to be developed and chosen based on the desired end point and general goal. Methods involving human subjects are probably of greatest value, although inter-individual variability is likely to be significant. Thus, even with the optimum method, bioavailability values for any given product are likely to be best represented by a range, rather than a single value. Increased availability and use of stable isotopes is likely to aid in the clarification of this issue of growing nutritional importance.

\section{References}

Blomhoff R, Green MH, Green JB, Berg T \& Norum KR (1991) Vitamin A metabolism: new perspectives on absorption, transport and storage. Physiology Reviews 71, 951-990. 
Bone RA, Landrum JT, Fernandez L \& Tarsis SL (1988) Analysis of the macular pigment by HPLC: retinal distribution and age study. Investigative Ophthalmology and Visual Science 29, 843-849.

de Pee S \& West C (1996) Dietary carotenoids and their role in combating vitamin A deficiency: a review of the literature. European Journal of Clinical Nutrition 50, Suppl. 3, S38-S53.

Gartner C, Stahl W \& Sies H (1997) Lycopene is more available from tomato paste than from fresh tomatoes. American Journal of Clinical Nutrition 66, 116-122.

Hammond BR, Johnson EJ, Russell RM, Krinsky NI, Yeum KJ, Edwards R \& Snodderly DM (1997) Dietary modification of human macular pigment density. Investigative Ophthalmology and Visual Science 38, 1795-1802.

Haskell MJ, Handelman GJ, Peerson JM, Jones AD, Rabbi MA, Awal MA, Wahed MA, Dilip M \& Brown KH (1997) Assessment of vitamin A status by the deuterated-retinol-dilution technique and comparison with hepatic vitamin A concentration in Bangladeshi surgical patients. American Journal of Clinical Nutrition 66, 67-74.

Mayne ST \& Parker RS (1989) Antioxidant activity of dietary canthaxanthin. Nutrition and Cancer 12, 225-236.

Miller NJ, Sampson J, Candeias L.P, Branley PM \& Rice-Evans CA (1996) Antioxidant activities of carotenes and xanthophylls. FEBS Letters 384, 240-246.

Novotny JA, Ducker SR, Zech LA \& Clifford AJ (1995) Compartmental analysis of the dynamics of $\beta$-carotene in an adult volunteer. Joumal of Lipid Research 36, 1825-1838.

Olson JA (1987) Recommended dietary intakes (RDI) of vitamin A in humans. American Journal of Clinical Nutrition 45, 704-716.

O'Neill ME \& Thurnham DI (1998) Intestinal absorption of $\beta$ carotene, lycopene and lutein in men and women following a standard meal: response curves in the triacylglycerol-rich lipoprotein fraction. British Journal of Nutrition 79, 149-159.
Ong DE (1994) Cellular transport and metabolism of vitamin A: roles of the cellular retinoid-binding proteins. Nutrition Reviews 52, S24-S31.

Parker RS (1996) Absorption, metabolism and transport of carotenoids. FASEB Journal 10, 542-551.

Parker RS (1997) Bioavailability of carotenoids. European Journal of Clinical Nutrition 51, Suppl. 1, S86-S90.

Parker RS, Brenna JT, Swanson JE, Goodman KJ \& Marmor B (1997) Assessing metabolism of $\beta-\left[{ }^{13} \mathrm{C}\right]$ carotene using highprecision isotope ratio mass spectrometry. Methods in Enzymology 282, 130-140.

Parker RS, Swanson JE, Marmor B, Goodman KJ, Spielman AB, Brenna JT, Viereck SM \& Canfield WK (1993) Study of $\beta$ carotene metabolism in humans using $13 \mathrm{C}$ - $\beta$-carotene and high precision isotope ratio mass spectrometry. Annals of the New York Academy of Sciences 691, 86-95.

Rock CL, Lovalvo JL, Ementiser C, Ruffin MT, Flatt SW \& Schwartz SJ (1998) Bioavailability of $\beta$-carotene is lower in raw than in processed carrots and spinach in women. Journal of Nutrition 128, 913-916.

Swanson JE \& Parker RS (1996) Biological effects of carotenoids in humans. In Handbook of Antioxidants, pp. 337-367 [L Packer and E Cadenas, editors]. New York, NY: Marcel Dekker Inc.

Traber MG, Diamond SR, Lane JC, Brody RI \& Kayden HJ (1994) $\beta$-Carotene transport in human lipoproteins. Comparisons with $\alpha$-tocopherol. Lipids 29, 665-669.

van Vliet T (1996) Absorption of $\beta$-carotene and other carotenoids in humans and animal models. European Journal of Clinical Nutrition 50, Suppl. 3, S32-S37.

van Vliet T, Schreurs WHP \& van den Berg H (1995) Intestinal $\beta$ carotene absorption and cleavage in men: response of $\beta$-carotene and retinyl esters in the triglyceride-rich lipoprotein fraction after a single oral dose of $\beta$-carotene. American Journal of Clinical Nutrition 62, 110-116. 